\title{
Moluscos gasterópodos terrestres como indicadores de recuperación de bosque en los paisajes del Sur y Norcentro de Nicaragua
}

\author{
Marlon Sotelo*, Adolfo López, S.J. **, Marvin A. Tórrez ${ }^{* * *}$ y \\ Wayne J. Arendt ${ }^{* * * *}$
}

Recibido: septiembre de 2015 / Aceptado: octubre de 2015

Los moluscos terrestres son un grupo altamente diverso cuyo trabajo investigativo en Nicaragua ha sido mayoritariamente en la vertiente del Pacífico de Nicaragua. Evaluar el potencial de estas especies como indicadores de la perdida de la masa boscosa así como de su recuperación ayudará el impacto de las actividades humanas en esta biota de escasa movilidad. La colectas de conchas y especímenes fué realizada en bosques húmedos, nubosos y secos en los departamentos de Rivas y Jinotega en hábitats específicos dentro de la matrix del paisaje. Las identificaciones a posteriori fueron hechas para evitar el sesgo de identificaciones en especies de taxonomía debatible. Los hábitats forestales continuos mostraron valores mayores en biodiversidad que otros hábitats dentro de la matrix, mostrando que existe fidelidad de hábitat para la comunidad de caracoles en general. Esta alta fidelidad, muestra la importancia de este grupo cuando tratamos de obtener especies de fidelidad alta, ayudando a explicar cómo la perdida de bosque afecta a la fauna.

Palabras clave: moluscos terrestres / indicador / recuperación de bosque / sensible

Consultor USDA Forest Service, International Institute of Tropical Forestry/Paso Pacífico, Carretera a Masaya Km 12.4, Residencial Villas del Prado, Casa No. 7, Managua, Nicaragua. Correo electrónico: marlon@pasopacifico.org

** Museo de Ciencias Naturales, Universidad Centroamericana, Rotonda Rubén Darío 150 mts. al oeste, Apdo.69, Managua, Nicaragua. Correo electrónico: alosi@ns.uca.edu.ni.

*** Estación Biológica Juan Roberto Zarruck, Universidad Centroamericana, Rotonda Rubén Darío 150 mts. al oeste, Apdo. 69, Managua, Nicaragua. Correo electrónico: mtorrez@ns.uca.edu.ni.

${ }^{* * * * *}$ USDA Forest Service, International Institute of Tropical Forestry, Sabana Field Research Station, HC 2 Box 6205, Luquillo 00773, Puerto Rico. Correo electrónico: warendt@fs.fed.us 


\section{Introducción}

La fauna de moluscos continentales de la región del Pacífico de Nicaragua ha sido bien estudiada desde el punto de vista taxonómico (Pérez, 1999; Pérez \& López 1999). Estos autores han realizado un inventario detallado de los gasterópodos continentales de esta región del país utilizando el método de cartografiado UTM, así como una caracterización taxonómica completa de algunas de las especies más relevantes de la zona. En Nicaragua, estudios cuantitativos de la diversidad en el nivel de comunidades, así como caracterizaciones del hábitat de las especies, se abordan mayormente a nivel de vertebrados (Harvey et al., 2006; Vílchez et al., 2007; Arendt, Tórrez \& Vílchez, 2012), sin embargo algunos estudios multitaxa han incluido a los moluscos en sus análisis (Pérez, et al., 2005, 2006) y de igual manera, Pérez, Sotelo, Arana y López (2008) y Pérez y Sotelo (2013) hacen un análisis detallado de esta taxa a nivel de uso de hábitat.

Las actividades humanas que tienen incidencias edáficas, muestran influencia en la biodiversidad del sotobosque (Clark, Clark, Sandoval \& Castro, 1995; Clark, Palmer \& Clark, 1999). Algunas prácticas inadecuadas y procedimientos agropecuarios, provocan a largo plazo desestabilización ecológica y transformación de las propiedades químicas, físicas y biológicas del suelo, afectando la calidad de la materia orgánica, su $\mathrm{pH}$, la temperatura, la humedad, la textura, la porosidad, y la cobertura vegetal del suelo. Estas transformaciones provocan desequilibrios, pérdidas o cambios en la composición de las comunidades edáficas. Por lo tanto, la presencia y el balance de algunos grupos de la fauna edáfica, como los gasterópodos terrestres, pueden ser utilizados como indicadores potenciales del grado de perturbación y/o recuperación del suelo.

En el presente trabajo se estudiaron las comunidades de moluscos gasterópodos continentales en dos áreas geográficas de Nicaragua, Jinotega en el Norte y Rivas en el Sur, donde se conoció el estado actual de las comunidades y cuáles especies están respondiendo de manera negativa a la pérdida de hábitat. Las especies consideradas indicadoras son importantes herramientas para la determinación del grado de afectación que sufre un ecosistema, ya sea causado por el hombre o por causas naturales (Lindenmayer, Margulies \& Botkin, 2000), por lo que esperamos que esta propuesta ayude a dilucidar cuáles pueden ser potencialmente usadas.

\section{Materiales y métodos}

\section{1. Área de estudio}

El área de estudio se localiza en dos zonas geográficas de Nicaragua: Jinotega en el Norte y Rivas en el Sur (Figura 1). En Jinotega se estudiaron tres localidades,

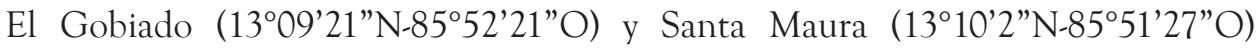
ubicadas dentro de la Reserva Natural Datanlí-El Diablo y la Reserva Silvestre Privada El Jaguar (N13⒕638'; 8603.177’W). En Rivas se estudiaron cuatro fincas en tres localidades diferentes, de la siguiente manera: Finca Isla Vista y Finca Sierra Serena en el municipio de Cárdenas (11¹3’29” N-85³3’9”O), Finca Guadalupe 


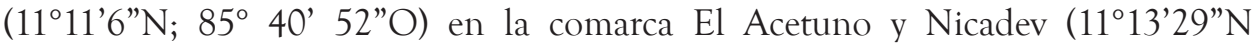
$\left.85^{\circ} 48^{\prime} 4^{\prime \prime} O\right)$ en Escamequita.

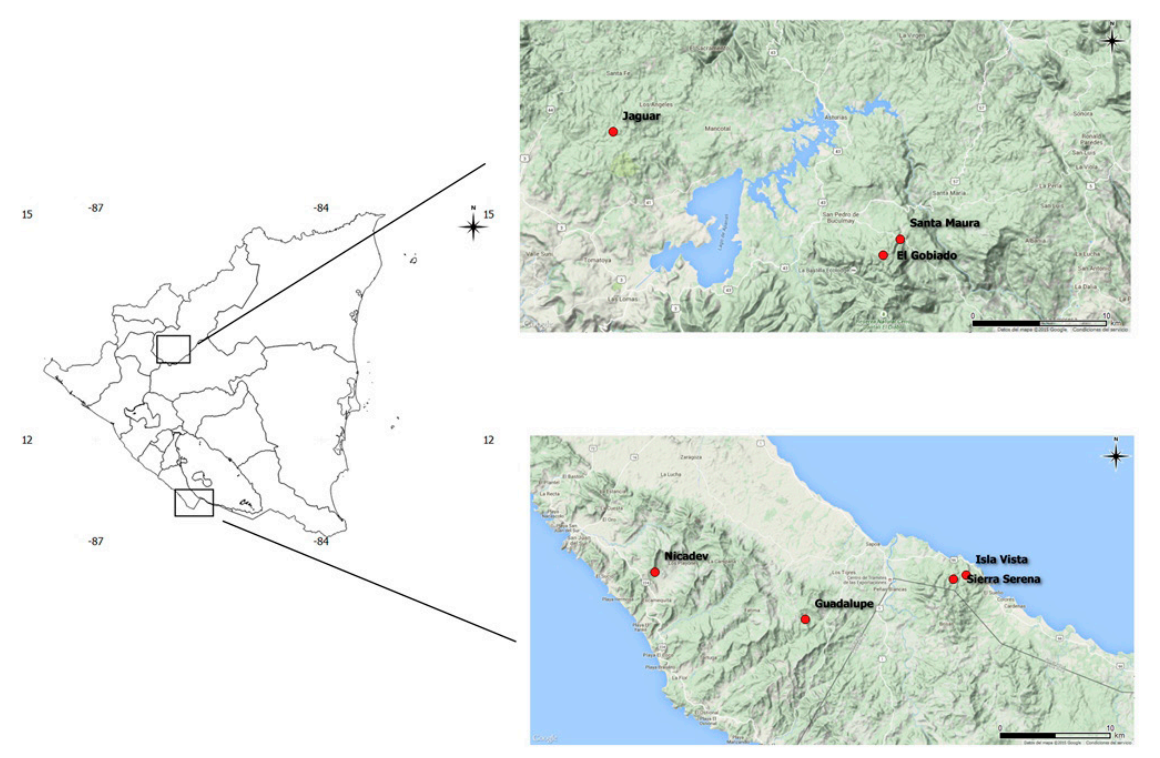

Figura 1. Sitios de muestreo en la zona de Jinotega (superior derecha) y en la zona de Rivas (inferior derecha).

Los datos fueron recolectados en puntos de monitoreo distribuidos en cinco tipos de formaciones vegetales, que son: tacotal, cafetal, hábitat abierto, bosque ripario y bosque secundario. El muestreo se efectuó mediante el levantamiento de rocas, revisión ocular de la hojarasca y/o el mantillo, corteza y oquedades de árboles, troncos, etc. En cada punto se recolectó en una bolsa plástica debidamente etiquetada con la fecha, el código del hábitat y la localidad, una muestra de mantillo de un área aproximada de $1 \times 1 \mathrm{~m}$., con el objetivo de no pasar por alto los microgasterópodos.

Toda la separación del material recolectado se realizó a mano con la ayuda de pinzas entomológicas y de un microscopio estereoscopio NIKON SMZ-10A con cámara clara. Los ejemplares encontrados se depositaron en cápsulas de gel debidamente etiquetadas y luego en tubos de cristal debidamente etiquetados. Todos los gasterópodos identificados se depositaron en la colección del Centro de Malacología y Diversidad Animal de la Universidad Centroamericana.

\subsection{Análisis taxonómico}

Los caracteres utilizados para la identificación de los especímenes fueron de tipo conquiológico, que consiste en el estudio de la morfología de la concha, su estructura, ornamentación y algunas otras características externa visibles. Para ello se emplearon descripciones, fotografías y dibujos procedentes de la literatura especializada y de la colección de fotos del Centro de Malacología. Además, en algunos casos, se hicieron comparaciones con los ejemplares ya identificados de la colección del Centro de Malacología y Diversidad Animal de la UCA. 


\subsection{Análisis de la información}

Se hizo comparaciones entre los hábitats a través de pruebas tipo ANOVA. Los supuestos de normalidad y homogeneidad de varianza fueron probados a través del análisis de los residuos y los predichos de los modelos de ANOVA, antes de comparar los hábitats, todo esto en el programa Infostat (Di Rienzo et al., 2011). El índice de Jaccard para determinar la similitud entre los hábitats y entre las localidades, y el índice de Equitatividad de Pielou, fueron analizados a través del programa Biodiversity Pro (McAleece, 1997).

\section{Resultados}

Se recolectó un total de 67 especies distribuidas en 20 familias y 41 géneros. De las 67 especies 42 se encontraron en las áreas estudiadas en el Norte y 40 en las áreas estudiadas en el Sur. El Gobiado y Santa Maura fueron las localidades que presentaron el número más alto de riqueza de especies $(S=32)$, seguido por $E 1$ Acetuno $(S=26)$ y Escamequita $(S=24)$.

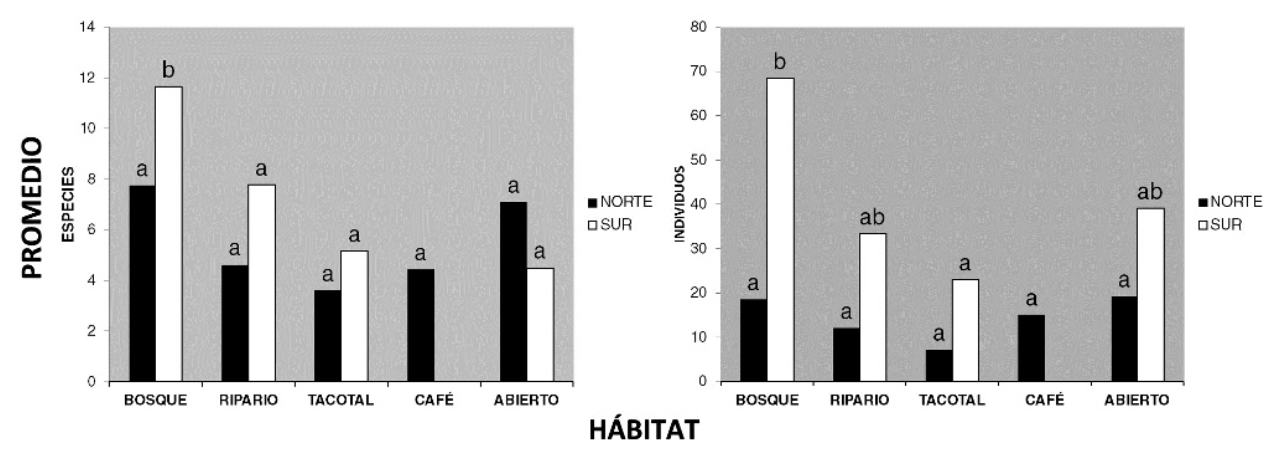

Figura 2. Promedio de las riquezas (Izq) y abundancias (Der), de moluscos tanto para las localidades de la zona norte como sur. Letras distintas muestran diferencias significativas $(\mathrm{p}<0.05)$

En el nivel de hábitat (Cuadro 1), el hábitat con mayor riqueza fue el bosque de la zona sur $(S=32)$ seguido del bosque de la zona norte $(S=27)$, aunque la zona sur tuvo más especies en su totalidad, la equitatividad de éstas fue menor en todos los casos excepto en el ripario (Cuadro 2). Para la zona norte las especies más comunes fueron Habroconus selenkai (9.8 \%), Drymaeus translucens (8.9\%), y Leptinaria guatemalensis $(8.1 \%)$, para los hábitats de la zona sur la especie más abundante fue Beckianum beckianum (28\%), seguido de Praticolella griseola (14\%). Los hábitat de la zona norte (Figura 2) no presentaron diferencias significativas en la riqueza $\left(\mathrm{F}_{4,34}=1.83, \mathrm{p}=0.1467\right)$ y en las abundancias $\left(\mathrm{F}_{4,34}=0.93, \mathrm{p}=0.4592\right)$, sin embargo en la zona sur (Figura 1), si se presentaron diferencias para ambas $\left(F_{3,26}=5.91, p<0.05\right)$ $\left(\mathrm{F}_{3,26}=16.96, \mathrm{p}<0.05\right)$. 
Cuadro 1. Valores de riqueza, abundancia e índice de equitatividad para los hábitat en estudio

Hábitat

\begin{tabular}{lll} 
& Diversidad & \\
\hline Abundancias & Riqueza & Equitatividad
\end{tabular}

Sur

$\begin{array}{llll}\text { BOSQUE } & 616 & 32 & 0.752 \\ \text { RIPARIO } & 300 & 24 & 0.824 \\ \text { TACOTAL } & 138 & 15 & 0.774 \\ \text { ABIERTO } & 235 & 12 & 0.555\end{array}$

Norte

$\begin{array}{lccc}\text { BOSQUE } & 148 & 27 & 0.853 \\ \text { RIPARIO } & 97 & 24 & 0.791 \\ \text { TACOTAL } & 36 & 15 & 0.877 \\ \text { CAFÉ } & 136 & 22 & 0.895 \\ \text { ABIERTO } & 173 & 26 & 0.846\end{array}$

Las tres localidades de la zona norte (Figura 3b) presentaron diferencias significativas en la riqueza $\left(\mathrm{F}_{2,36}=4.38, \mathrm{p}=0.019\right)$, al igual que las del sur (Figura 3a), $\left(\mathrm{F}_{2,27}=6.49, \mathrm{p}=0.005\right)$, sin embargo la abundancias para las localidades de la zona norte (Figura 3d) fueron similares $\left(\mathrm{F}_{2,36}=0.77, \mathrm{p}=0.4714\right.$ ), no así el sur (Figura 3c) donde las abundancias en la localidades variaron $\left(F_{2,27}=4, p=0.03\right)$.
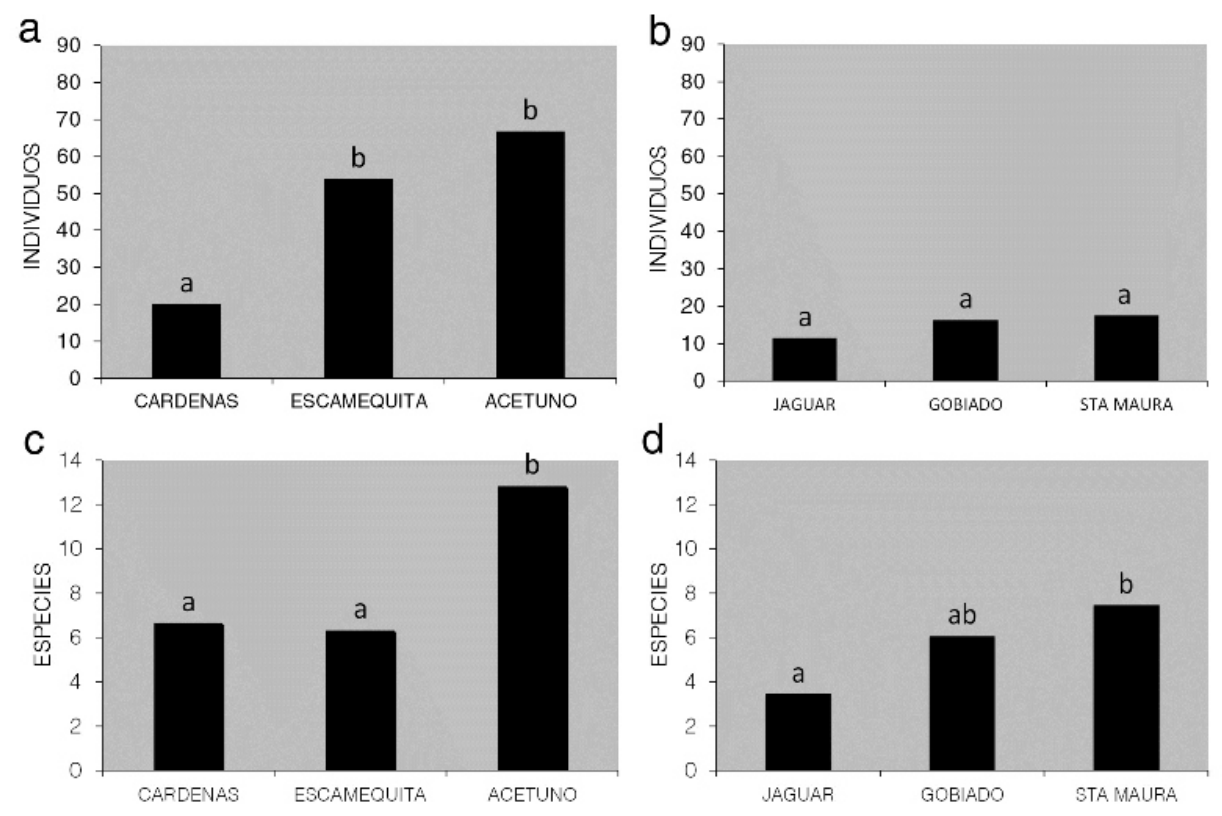

Figura 3. Promedios de abundancia $(a, b)$ y riqueza (c, d) en los comunidades en estudio 
La forma en que los hábitats se agruparon en el análisis de similitud fue diferente para ambas zonas (Figura 4). Mientras que en la zona norte se formó un agrupamiento donde las áreas antropizadas como el hábitat abierto y el cafetal muestran mayor afinidad con el bosque, en el sur el bosque se asoció únicamente con el otro hábitat con cobertura boscosa como lo es ripario. En ambas áreas los tacotales fueron los hábitats que mostraron menos similitud con los otros hábitats.
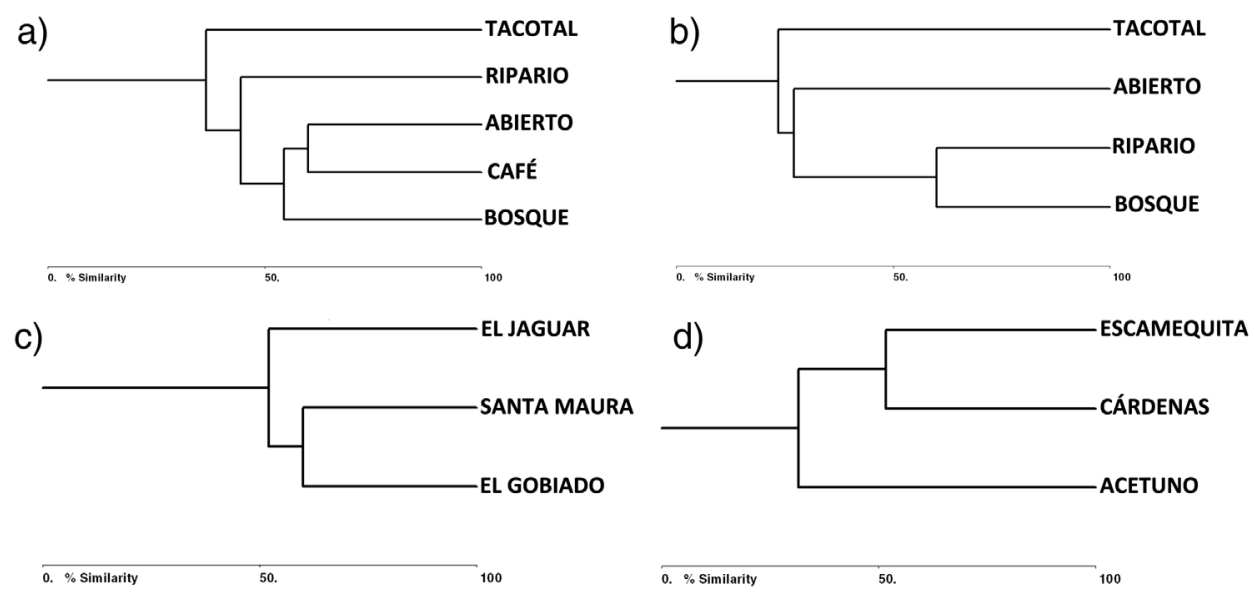

Figura 4. Dendrograma de similitud de comunidades utilizando el índice de Jaccard. a) Hábitat de la zona norte, b) hábitats de la zona sur; c) y d), localidades de norte y sur respectivamente

\subsection{Análisis de las especies indicadoras}

Se seleccionaron dos grupos de especies indicadoras. El primer grupo es el de aquellas especies encontradas exclusivamente en hábitats conservados (Cuadro 2) y cuya presencia está asociada a sitios que no tienen una historia reciente de antropización. En segundo lugar aquellas especies cuya presencia refleja algún gradiente que se puede considerar predictible, ya que ésta disminuye de manera gráfica, como disminuye el grado de conservación del hábitat. 
Cuadro 2. Especies que se encontraron únicamente en hábitats con cobertura boscosa durante el estudio

\begin{tabular}{|c|c|c|c|}
\hline \multirow{9}{*}{ Norte } & Especie & BOSQUE & RIPARIO \\
\hline & & $n=148$ & $n=97$ \\
\hline & Beckianum beckianum & & 7 \\
\hline & Drymaeus attenuatus & 4 & 4 \\
\hline & Euglandina obtusa & 2 & \\
\hline & Helicina oweniana & 3 & \\
\hline & Helix trigonostoma & 4 & \\
\hline & Salasiella guatemalensis & 1 & 1 \\
\hline & Xenodiscula taintori & & 1 \\
\hline \multirow[t]{18}{*}{ Sur } & & $n=616$ & $n=300$ \\
\hline & Chanomphalus pilsbryi & 20 & 25 \\
\hline & Drepanostomella pinchoti & 10 & 12 \\
\hline & Drymaeus alternans & 19 & 10 \\
\hline & Euglandina gigantea & 1 & \\
\hline & Gastrocopta geminidens & & 2 \\
\hline & Guppya gundlachi & 19 & \\
\hline & Miradiscops opal & 18 & 2 \\
\hline & $\begin{array}{l}\text { Neocyclotus dysoni } \\
\text { nicaraguense }\end{array}$ & 12 & 9 \\
\hline & Opeas pumilum & 6 & \\
\hline & Pupisoma dioscoricola & 2 & 3 \\
\hline & Sterkia antillensis & 6 & 1 \\
\hline & Thysanophora caecoides & 2 & \\
\hline & Thysanophora crinita & 7 & \\
\hline & Thysanophora hornii & 9 & \\
\hline & Thysanophora plagioptycha & 6 & \\
\hline & Trichodiscina coactiliata & 9 & 2 \\
\hline & Xenodiscula taintori & 14 & 11 \\
\hline
\end{tabular}

La especie Cecilioides consobrinus (Figura 5) fue la única que presentó diferencias significativas $\left(\mathrm{F}_{3,5}=13.3 \mathrm{p}=0.07\right)$. Esta especie fue encontrada únicamente en la zona sur, principalmente en el Acetuno y Escamequita, no encontrándose en Cárdenas. 


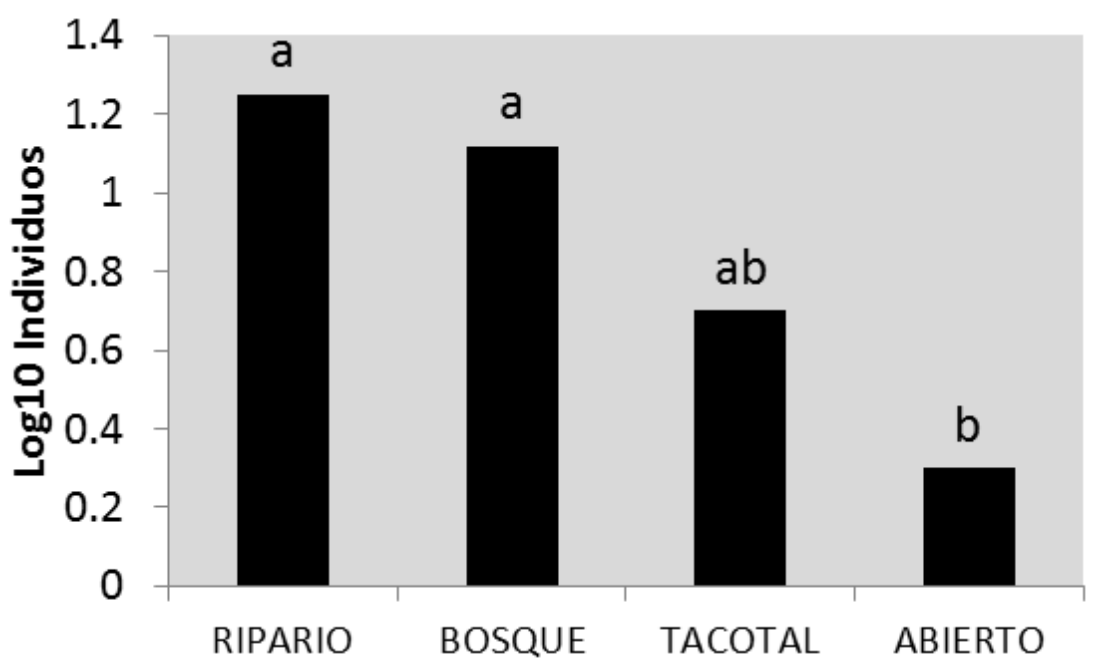

Figura 5. Promedio de observaciones de la especie Ceciliodes consobrinus, mostrando su disminución de los hábitats mejor conservados a los menos conservados

\section{Discusión}

En ambas zonas de estudio las áreas de bosque mostraron mayor riqueza de especies, siendo esto consistente con estudios previos (Pérez et al., 2006, 2008), pero no así en Pérez y Sotelo (2013), donde el hábitat con mayor riqueza fue el cafetal con sombra. La abundancia de individuos presentó igual comportamiento en la zona sur, donde las áreas de bosque presentaron mayor abundancia con respecto a los otros hábitats. Ambos criterios muestran cómo este grupo aumenta en biodiversidad según la estructura boscosa, ya que tanto en riqueza, como en abundancia la comunidad de moluscos es mayor en hábitats mejor conservados, específicamente en el caso del Paso del Istmo, además que sumado a esto al comparar los hábitats se observa que el índice de equitatividad es mayor en áreas con cobertura boscosa en relación con las que no tienen cobertura.

Las localidades mostraron resultados en los que tanto El Acetuno (Paso del Istmo) como finca Santa Maura (Zona Norte) son lugares que presentaron mayor riqueza de especies. Ambos comparten el hecho de ser áreas boscosas continuas relativamente grandes (> $100 \mathrm{Ha}$ ), lo que posibilita que alberguen una mayor cantidad de especies. Es de notar que los hábitats de la zona norte, de acuerdo a la composición de especies de moluscos mostraron mayor similitud entre hábitats abiertos y cafetales, uniéndose luego al bosque, siendo lo hábitats más disimiles el bosque ripario y los tacotales. No obstante, en el sur los hábitats más conservados, como los riparios y bosques formaron un solo grupo, uniéndose luego el hábitat abierto y tacotal por su similitud. Los hábitats de cafetales de la zona norte presentan una presencia homogénea de árboles, los cuales proveen de microhábitats óptimos para la presencia de estos moluscos ya que la presencia de árboles es una variable importante en la diversidad de este grupo (Oke \& Chokor, 2009) y, según la historia del sitio, se sabe que los árboles remanentes son de las áreas boscosas que cambian 
a cafetales. Por esto es probable que especies presentes en el bosque logren persistir con éxito en el cafetal cuando la cobertura boscosa no ha sido removida en un $100 \%$, además que se resalta el hecho de que los hábitats de cafetal y áreas abiertas dentro del muestreo están dentro de una matriz mixta con remanentes de bosque relativamente cercanos $(\square 25 \mathrm{~m})$.

En el norte las localidades mostraron mayor afiliación según la cercanía geográfica (Figura 4), sin embargo en el sur no fue así, donde la afiliación fue entre más distantes (Escamequita y Cárdenas). Estos hábitats, aparte de estar alejados, no comparten afinidad ecológica natural por estar en zonas climáticas diferentes (INETER, 2005). Pero al hacer una observación detallada de los datos se observó que comparten epsecies generalistas como Bulimulus corneus, y Praticolalla griseola, las cuales son especies generalistas ampliamente distribuidas (Pérez \& López, 2002) pero, aparte de eso, se logra notar que muchas especies fueron únicas para la comunidad el Acetuno, haciendo por lo tanto que la riqueza de esta localidad sea más singular con respecto a las otras.

Los moluscos terrestres que mostraron estar presentes en hábitats específicos (Cuadro 2) son un primer acercamiento para saber cuáles especies pueden considerarse hábitat-dependientes y por lo tanto demostrar cuándo un hábitat ha alcanzado cierto grado de madurez. Sin embargo, esperamos que mayor investigación al respecto pueda mostrar si estas observaciones son o no casuales.

El uso de un grupo de especies, como los moluscos que presentan una escasa movilidad, debe de valorarse como útil en la determinación del estado de los hábitats, dado que una vez que se ha cambiado un uso de suelo en un $100 \%$, los individuos que habiten estas zonas tienen poco chance de encontrar refugio o recolonizar hábitats aledaños. Por lo tanto, la carencia o disminución de estas especies es un indicador de la composición original de un área, que cuenta con con mayor fidelidad que en el caso de especies que presentan mayor capacidad de movilidad.

\section{Agradecimientos}

Este trabajo no hubiera sido posible sin la invaluable colaboración de la ONG Paso Pacífico y el gobierno de los Estados Unidos a través de la agencia de los Estados Unidos para el Desarrollo Internacional (USAID) y el Instituto Internacional de Dasonomía Tropical del Servicio Forestal Federal de los Estados Unidos, bajo el proyecto Conservación y Turismo Sostenible en Cuencas Críticas. Es de especial interés mostrar nuestro agradecimiento al señor Jerry Bauer, cuya gestión hace posible culminar este tipo de investigaciones. Manifestamos nuestro sincero agradecimiento a los propietarios, quienes brindaron apoyo en el acceso a las fincas y en abrirnos las puertas a tal importante área del Paso del Istmo. También agradecemos el trabajo arduo, empeño, y entusiasmo de los técnicos de campo: Iván Ramírez Barrera, Fredder Antonio Ortega Sequeira, Juan Cruz Gámez, Henry Julián López, Jorge Hernández, Elmeyer Guevara y los guías locales, ya que han sido invaluables para nuestro trabajo de campo gracias a su ayuda y apoyo en la investigación. Parte de estas investigaciones se llevó a cabo en cooperación con la Universidad de Puerto Rico. 


\section{Referencias bibliográficas}

Arendt, W.J., Tórrez, M.A., \& Vílchez, S.J. (2012). Diversidad de Aves en Agropaisajes en la Región Norte de Nicaragua. Ornitología Neotropical, 23, 113-131.

Clark, D.A., Clark, D.B., Sandoval, R., \& Castro, M.V. (1995). Edaphic and Human Effects on Landscape-Scale Distributions of Tropical Rain Forest Palms. Ecology, 76, 2581-2594.

Clark, D.B., Palmer, M.W., \& Clark, D.A. (1999). Edaphic Factors and the LandscapeScale Distribution of Tropical Rain Forest Trees. Ecology, 80, 2662-2675.

Di Rienzo J.A., Casanoves F., Balzarini M.G., Gonzalez L., Tablada M. \& Robledo C.W.(2011). InfoStat versión 2011. Grupo InfoStat, FCA, Universidad Nacional de Córdoba, Argentina. URL http://www.infostat.com.ar.

Harvey, C,A., Medina, A., Sánchez, D.M., Vílchez, S., Hernández, B., Saenz, J.C., Maes, J.M., Casanoves, F. \&, Sinclair, F.L.(2006). Patterns of Animal Diversity in Different Forms of Tree Cover in Agricultural Landscapes. Ecological Applications, 16, 1986-1999.

INETER. (2005). Clasificación climática según Köppen: Período 1971-2000. [Mapa a escala 1:12500]. Managua, Nicaragua: Autor. Recuperado de http://webgeofisica.ineter.gob.ni/mapas/Nicaragua/clima/atlas/Clasificacion\%20 Climatica/Clasificacion_Climatica_Koppen.jpg.

Lindenmayer, D., Margulies, C., \& Botkin, D. (2000) Indicators of Biodiversity for Ecologically for Sustainable Forest Management. Conservation Biology, 14, 941-950.

McAleece, N.(1997). BioDiversity Professional Version 2.0. The Natural History Museum \& The Scottish Association for Marine Science.

Oke, O.C., \& Chokor, J.V.(2009). Lands Snail Populations in Shade and Full-Sun Cocoa Plantations in South Western Nigeria, West Africa. African Scientist, 10, 19-29.

Pérez, A.M. (1999). Estudio Taxonómico y Biogeográfico Preliminar de la Malacofauna Continental (Mollusca: Gastropoda) del Pacífico de Nicaragua. Tesis Doctoral. Universidad del País Vasco, España.

Pérez, A.M. \& López, A. (1999). Estudio Taxonómico y Biogeográfico Preliminar de la Malacofauna Continental (Mollusca: Gastropoda) del Pacífico de Nicaragua (19951998). Cuaderno de Investigación. Managua: UCA Publicaciones.

Pérez, A. M., \& López, A. (2002). Atlas de los Moluscos Gasterópodos Continentales del Pacífico de Nicaragua. Managua: UCA Publicaciones.

Pérez, A.M. \& Sotelo, M. (2013). Species Richness of Continental Gastropod Mollusk from a Coffee Plantation in Jinotega, Northern Nicaragua: a first approach. Cuadernos de Investigación UNED, 5, 17-23.

Pérez, A.M., Sotelo, M., Arana, I., \& López, A.(2008). Diversidad de Moluscos Gasterópodos Terrestres en la Región del Pacífico de Nicaragua y sus Preferencias de Hábitat. Revista de Biología Tropical, 56, 317-332.

Pérez, A. M., Sotelo, M., Ramírez, F., Ramírez, I., López, A. \& Siria, I. (2005). Composición y Diversidad de las Comunidades de Aves Moluscos y Plantas Asociadas con Sistemas Silvopastoriles de Matiguás y Río Blanco, Dpto. de 
Matagalpa, Nicaragua. Encuentro, (71), 136-165.

Pérez, A. M., Sotelo, M., Ramírez, F., Ramírez, I., López, A., Siria, I. (2006). Conservación de la Biodiversidad en Sistemas Silvopastoriles de Matiguás y Río Blanco (Matagalpa, Nicaragua) . Ecosistemas, (3), 1-16.

Vílchez, S.J., Harvey, C., Sánchez-Merlo, D., Medina, A., Hernández, B. \& Taylor, R. (2007). Diversidad y Composición de Aves en un Agropaisaje de Nicaragua. En C. Harvey \& J. C. Sáenz, (Eds). Evaluación y Conservación de Biodiversidad en Paisajes Fragmentados de Mesoamérica. (pp. 547-576) (1ed.). Santo Domingo de Heredia, CostaRica: Instituto Nacional de Biodiversidad, INBio. 